\title{
Research on Wavelet Threshold Denoising Method for UWB Tunnel Personnel Motion Location
}

\author{
Ning Liu ${ }^{(D)}{ }^{1}$ Ranqiao Zhang, ${ }^{1}$ Zhong Su ${ }^{D},{ }^{1,2}$ Guodong Fu, ${ }^{1,3}$ and Jingang $\mathrm{He}^{4}$ \\ ${ }^{1}$ Beijing Key Laboratory of High Dynamic Navigation Technology, Beijing Information Science \& Technological University, \\ Beijing 100101, China \\ ${ }^{2}$ School of Automation, Beijing Institute of Technology, Beijing 100081, China \\ ${ }^{3}$ Beijing Dewei Chuang Ying Technology Co., Ltd, Beijing 100081, China \\ ${ }^{4}$ China Communications Information Center Co., Ltd, Beijing 100085, China \\ Correspondence should be addressed to Ning Liu; ning.liu@bistu.edu.cn
}

Received 27 August 2019; Revised 3 November 2019; Accepted 19 November 2019; Published 28 February 2020

Academic Editor: Konstantinos Karamanos

Copyright (c) 2020 Ning Liu et al. This is an open access article distributed under the Creative Commons Attribution License, which permits unrestricted use, distribution, and reproduction in any medium, provided the original work is properly cited.

In the process of tunnel construction, the problems of strong sealing, inconvenient communication, and harsh environment pose a serious threat to the personal safety of construction workers. Therefore, personnel positioning technology has important application value in tunnel safety construction. A special environment for tunnel personnel positioning and the ultrawideband (UWB) positioning system are affected by personnel movement, which leads to the problem of lowering positioning accuracy. A wavelet threshold denoising method for motion positioning of people in tunnels is proposed. The positioning algorithm of the method adopts a three-sided positioning algorithm based on symmetric double-sided two-way ranging. The wavelet analysis is used to decompose the motion signal of the personnel in the tunnel, and the low frequency coefficient and high frequency coefficient of the signal are decomposed to determine the influence of the motion noise of the personnel on the UWB positioning. The soft threshold function and the hard threshold function are, respectively, selected to perform wavelet threshold denoising on the motion positioning result in the tunnel. According to the denoising effect, the db5 wavelet 5-layer decomposition, under the heuristic threshold estimation criterion, the soft threshold function denoising is the best denoising method. The verification by the positioning experiment shows that the method is suitable for tunnel personnel positioning. The wavelet threshold denoising method can weaken the influence of outliers in the motion positioning of UWB personnel and improve the positioning accuracy.

\section{Introduction}

In recent years, with the continuous development of the economy, more urgent requirements have been put forward on the transportation network. Building a modern transportation network is an inevitable requirement of economic construction and social development. Therefore, tunnel construction of roads, railways, and subways is an important component of the transportation network. The safety of tunnel construction has become a hot issue [1].

In the process of tunnel construction, there are some problems due to the strong sealing, inconvenient communication, and bad environment. In the event of a landslide or other security incident, due to the existing communication conditions inside and outside the tunnel and the limitations of the communication mechanism, it is difficult to grasp the distribution of personnel under the tunnel during rescue, resulting in low efficiency of rescue and difficulties in search and rescue [2]. Therefore, real-time safety monitoring of the tunnel construction site and obtaining specific location information of each construction person are of great significance for reducing accident losses. The precise positioning of the tunnel cannot be ignored.

At present, the technologies commonly used for personnel positioning under tunnels include Radio Frequency Identification (RFID) positioning technology [3, 4], Zigbee positioning technology [5], Wi-Fi positioning technology [6], and UWB positioning technology [7]. UWB technology 
first appeared in the 1960s, and its initial application was limited to military, disaster rescue search, radar positioning, and ranging. Ultrawideband transmission power of $-41.3 \mathrm{dBm} / \mathrm{MHz}$ and the frequency range of 3.1 to $10.6 \mathrm{GHz}$ were established by the Federal Communications Commission (FCC) in the United States [8].

UWB positioning technology has advantages that other positioning technologies cannot match. It has strong antiinterference ability, high transmission rate, high security, strong penetrating ability, high multipath resolution, and relatively simple system structure $[9,10]$. Communication with nanosecond narrow pulses does not require carrier signal modulation, and its positioning accuracy can reach centimeter level [11]. These advantages make UWB positioning technology a better choice for high-precision wireless positioning of tunnels.

In the 1990s, SCHOLTZ RA first proposed the use of impulse pulses for time-hopping modulation in multipleaccess communication systems [12]. This landmark paper opened the first step in systematic research on UWB theory. Since then, UWB technology has become a hot spot in current wireless communication research $[13,14]$. The application of UWB positioning technology in tunnel personnel positioning also faces many challenges, such as signal acquisition in real environment, multiuser mutual interference, multipath effect, and non-line-of-sight propagation. Savic et al. [15] studied non-line-of-sight recognition based on the UWB positioning system in tunnels by analyzing channel propagation parameters. Matteo Ridol et al. [16] analyzed the effects of tag placement and human motion patterns on positioning accuracy and communication reliability and designed two algorithms, particle filter and Kalman filter, to further improve the accuracy of the localization algorithm.

In [17], the authors mentioned that the error in UWB positioning is mainly divided into low-frequency noise and high-frequency noise. The low-frequency noise needs to be processed by analysis and modeling, and the high-frequency noise can be processed by filtering and noise reduction, thereby effectively improving the accuracy of the positioning result. Peng [18] can use wavelet transform to distinguish the characteristics of the modulus maxima of signals and noise, improve the threshold function, and perform wavelet threshold denoising on pulse signals. Lin et al. [19] modeled multipath propagation and non-line-of-sight interference that affect indoor positioning accuracy, using wavelet analysis to denoise the ranging values, thereby improving positioning accuracy. Gong et al. [20] simulated the channel model and used wavelet denoising to eliminate non-line-ofsight (NLOS) error based on the traditional indoor positioning algorithm.

In the field of denoising, wavelet theory has been paid attention by many scholars. Wavelet denoising has been applied to one-dimensional signal and two-dimensional signal denoising [21-23] and achieved very good results. Specifically, the success of wavelet denoising method is mainly due to the following characteristics of wavelet transform [24]: (1) Multiresolution: the multiresolution method can well describe the nonstationary characteristics of the signal. (2) Low legitimacy: the sparse distribution of wavelet coefficients makes the entropy of the signal to reduce after wavelet transform. (3) Decorrelation: because the wavelet transform can decorrelate the signal and the noise can tend to whiten after the wavelet transform, the wavelet domain is more conducive to denoising than the time domain. (4) Base selection flexibility: wavelet transform can choose different wavelet basis functions according to different application situations and different research objects to obtain the best effect.

In this paper, we study the accuracy of static positioning and motion localization of the UWB tunnel personnel positioning system. According to the requirements and characteristics of tunnel location, the symmetric bilateral bidirectional ranging method is used and the three-way location algorithm based on time of arrival (TOA) is used. In the actual experiment, we found that due to the diversity and irregularity of the movement of the people, the noise of the motion was generated and the noise could not be completely avoided. When the UWB positioning system is used to locate the moving personnel, the positioning result may have abnormal jumping points and fluctuations, resulting in lower positioning accuracy and poor positioning reliability. We perform wavelet analysis on the motion positioning signal of the personnel in the tunnel and determine the influence of the motion noise of the personnel on the UWB positioning by separating the lowfrequency coefficient and the high-frequency coefficient of the signal. In this paper, wavelet threshold denoising can not only remove the noise in the system but also retain the important characteristics of the signal and has the ability to highlight the local characteristics of the signal in the time and frequency domain. The wavelet threshold denoising is used to denoise the motion positioning results. The soft threshold function and the hard threshold function are used, respectively, to determine the wavelet threshold denoising method which is more suitable for the motion location of people in the tunnel. The purpose of eliminating the abnormal positioning point and smoothing the positioning result is achieved.

The remainder of this paper is organized as follows. First, the principle of UWB ranging and positioning is outlined. Then, wavelet analysis is used to analyze the tunnel personnel positioning signal to determine the influence of personnel motion noise in the tunnel on UWB positioning. Next, the wavelet threshold denoising method and different threshold functions are introduced. Finally, the experiment and analysis are carried out, and the static positioning and motion positioning experiments are carried out in the tunnel, respectively, and the soft threshold function and the hard threshold function are compared, which is more suitable for the motion positioning of the personnel in the tunnel.

\section{Principle of UWB Ranging and Positioning}

There are three main methods for UWB positioning: angle of arrival (AOA), TOA, and time difference of arrival (TDOA). This paper uses the TOA-based methods for positioning. 
2.1. Principle of $U W B$ Ranging. The TOA positioning method first measures the time of flight (TOF) of the signal transmitted between each base station and the tag. Then, the TOF is multiplied by the propagation speed of the electromagnetic wave to calculate the distance from each base station to the tag. Therefore, the measurement of signal flight time becomes the key to ranging. One-Way Ranging (OWR), Two-Way Ranging (TWR), Single-Sided Asymmetric Two-Way Ranging (SSS-TWR), and Symmetric Double-Sided Two-Way Ranging (SDS-TWR) are the ranging protocols commonly used for TOA estimation.

The UWB positioning system selected in this paper uses the SDS-TWR method to measure the distance between the tag and the base station. This method is a two-way ranging technology, which can reduce the accuracy of the ranging accuracy caused by clock drift as much as possible. The principle of information exchange is shown in Figure 1.

First, device $A$ sends signal to device $B$, device $B$ receives the signal from device $A$ and sends signal to device $A$ after elapse of $t_{\text {reply }_{B}}$, and device $\mathrm{A}$ receives the signal sent by device $B$ and returns another signal to device $B$ after elapsed time $t_{\text {reply }_{A}}$.

The time when device A sends a signal to device A receiving a signal is indicated by $t_{\text {round }_{A}} ; t_{\text {round }_{B}}$ indicates the time when device $B$ sends a signal to device $B$ receiving a signal.

According to the time relationship shown in Figure 1, equation (1) can be obtained as follows:

$$
t_{\text {round } A}+t_{\text {tound } B}=4 \mathrm{TOF}+t_{\text {replyA }}+t_{\text {reply } B} \text {. }
$$

The TOF measurement is expressed as

$$
\mathrm{TOF}=\frac{t_{\text {round } A}+t_{\text {tound } B}-t_{\text {reply } A}-t_{\text {reply } B}}{4} .
$$

After adding the effects of device A and device B clock drift, the actual TOF is expressed as

$$
\text { TÔF }=\frac{\left(1+e_{A}\right)\left(t_{\text {round } A}-t_{\text {reply } A}\right)+\left(1+e_{B}\right)\left(t_{\text {tound } B}-t_{\text {reply } B}\right)}{4} \text {. }
$$

Therefore, the error of the transmission time of the wireless data packet in the air is

$$
\text { TÔF }- \text { TOF }=\frac{\left(e_{A}-e_{B}\right)}{2} \mathrm{TOF}+\frac{\left(e_{A}-e_{B}\right)\left(t_{\text {reply } B}-t_{\text {reply } A}\right)}{4} \text {. }
$$

Because under actual conditions, the value of TOF is much smaller than $t_{\text {reply }_{B}}$ and $t_{\text {reply }_{A}}$, the ranging error can be expressed as

$$
D_{\text {error }}=(\mathrm{TÔF}-\mathrm{TOF}) c=\frac{\left(e_{A}-e_{B}\right)\left(t_{\text {reply } B}-t_{\text {replyA }}\right)}{4} c .
$$

Although the SDS-TWR ranging method adds one information transmission than the conventional TWR ranging method, it can reduce the error caused by the crystal clock drift. At the same time, it does not require absolute

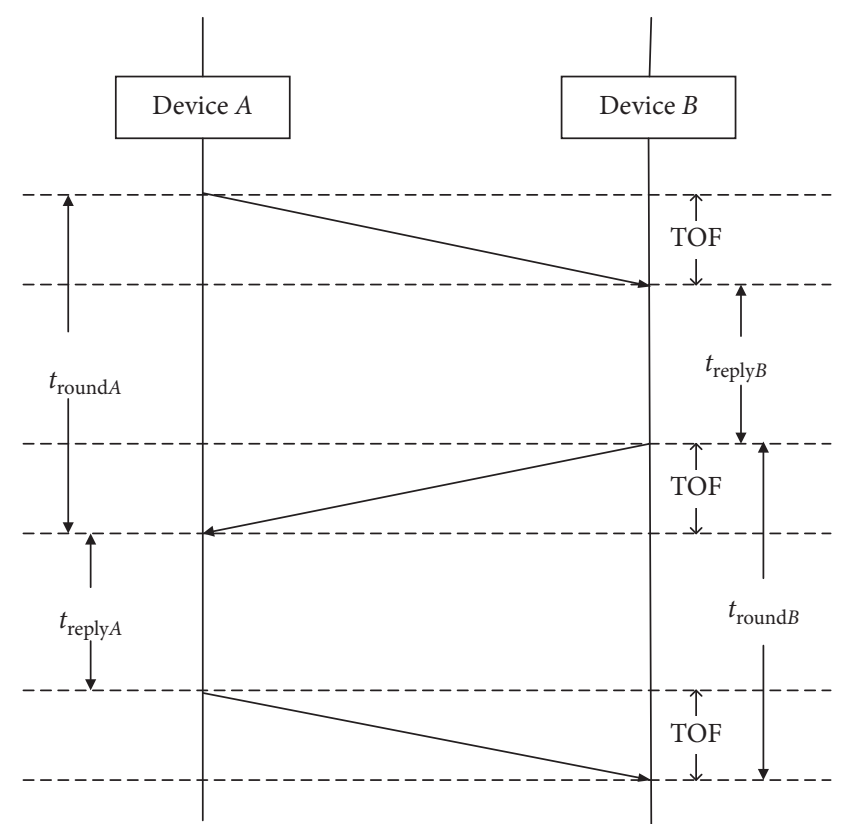

FIGURE 1: Schematic diagram of SDS-TWR.

synchronization of the signal sender and receiver clocks, which can greatly reduce the synchronization complexity caused by multitag access.

The propagation time of the signal from the positioning base station to the target tag is multiplied by the propagation speed of the radio in the air, and the distance $d$ from the positioning base station to the moving target tag can be obtained and the calculation method is as shown in:

$$
d=c \times \mathrm{TOF},
$$

where: $c$ is the propagation speed of the electromagnetic wave, and its value is $3 \times 108$, and the unit is $\mathrm{m} / \mathrm{s}$.

2.2. Principle of UWB Positioning. The TOA-based trilateration method first performs ranging and then uses the ranging value for positioning. Its mathematical model is shown in Figure 2.

The distances of the three positioning base stations $\mathrm{BS}_{i}\left(x_{i}, y_{i}\right)(i=1,2,3)$ to the tag $\mathrm{MS}(x, y)$ are, respectively, $d_{i}$. Assume that the error of the ranging value is zero. In this case, with the base station as the center and the ranging value as the radius, three circles can be obtained. The three circles have an intersection point, which is the position of the tag. The least squares method can be used to estimate the position of the tag. If two positioning base stations are used for positioning, there will be two intersection points, and the exact position of the label cannot be determined. Therefore, at least three base stations are selected for positioning based on TOA-based two-dimensional positioning.

Assuming that the coordinates of the tag are $(x, y)$, the coordinates of the $i$ positioning base station are $\left(x_{i}, y_{i}\right)$ and according to their geometric meaning, the relationship between them is as shown in the following equation: 


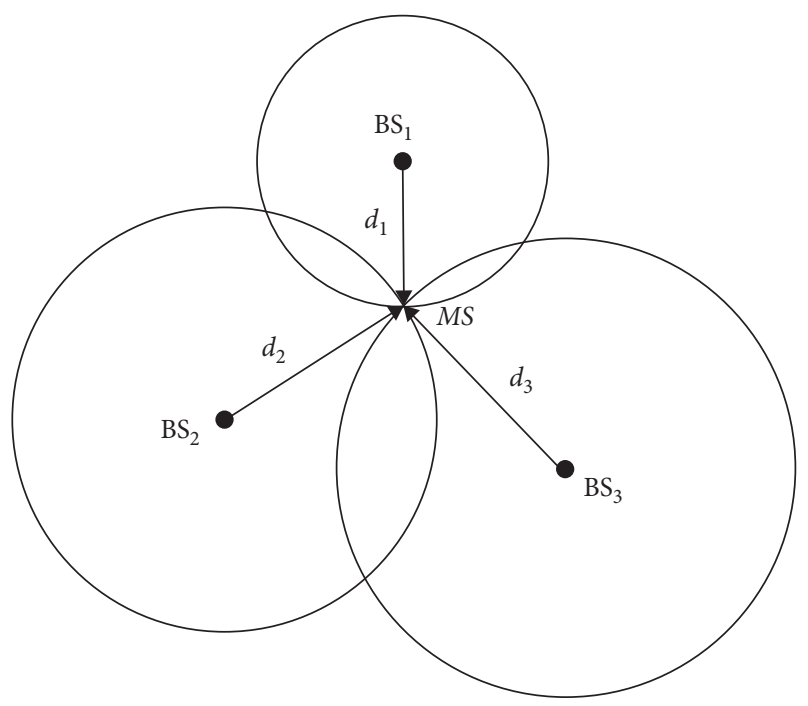

FIGURE 2: Schematic diagram of the TOA-based trilateration method.

$$
\left(x_{i}-x\right)^{2}+\left(y_{i}-y\right)^{2}=d_{i}^{2}, \quad i=1,2, \ldots, N
$$

Extending equation (7), using the first $n-1$ equations, and subtracting the $n$ equation, respectively, we obtain the linearized equation as follows:

$$
\begin{gathered}
{\left[\begin{array}{cc}
2\left(x_{1}-x_{N}\right) & 2\left(y_{1}-y_{N}\right) \\
\vdots & \vdots \\
2\left(x_{N-1}-x_{N}\right) & 2\left(y_{N-1}-y_{N}\right)
\end{array}\right]\left[\begin{array}{c}
x \\
y
\end{array}\right]} \\
\quad=\left[\begin{array}{c}
x_{1}^{2}-x_{N}^{2}+y_{1}^{2}-y_{N}^{2}+d_{N}^{2}-d_{1}^{2} \\
\vdots \\
x_{N-1}^{2}-x_{N}^{2}+y_{N-1}^{2}-y_{N}^{2}+d_{N}^{2}-d_{N-1}^{2}
\end{array}\right] .
\end{gathered}
$$

In equation (8), $A=\left[\begin{array}{cc}2\left(x_{1}-x_{N}\right) & 2\left(y_{1}-y_{N}\right) \\ \vdots & \vdots \\ 2\left(x_{N-1}-x_{N}\right) & 2\left(y_{N-1}-y_{N}\right)\end{array}\right]$, $X=\left[\begin{array}{c}x \\ y\end{array}\right]$, and $b=\left[\begin{array}{c}x_{1}^{2}-x_{N}^{2}+y_{1}^{2}-y_{N}^{2}+d_{N}^{2}-d_{1}^{2} \\ \vdots \\ x_{N-1}^{2}-x_{N}^{2}+y_{N-1}^{2}-y_{N}^{2}+d_{N}^{2}-d_{N-1}^{2}\end{array}\right]$.

Using the least squares method to solve equation (8), we can get

$$
X=\left(A^{T} A\right)^{-1} A^{T} b .
$$

where $X$ is the coordinate of the tag $(x, y)$.

\section{Analysis of the Positioning Signal of Tunnel Personnel}

Because when collecting the location signal of the personnel in the tunnel, there is no way to completely avoid the influence of noise signals caused by human movement. The final collected signal is always mixed with noise. This phenomenon will lead to large errors in the positioning result. Therefore, denoising the signal is an important problem in signal processing. Before starting the denoising process, wavelet analysis of the tunnel personnel motion localization signal is first performed. Wavelet analysis is a localized analysis of time and frequency. The signal is gradually multiscale, refined by telescopic translation. Finally, it has a higher frequency resolution and a lower time resolution in the low-frequency part, and a higher time resolution and lower frequency resolution in the high-frequency part.

3.1. Wavelet Analysis. Wavelet transform is a localized analysis of time (space) frequency, which gradually multiscales the signal (function) through the stretching and translation operation. Finally, it reaches the time division at high frequency, and the frequency is subdivided at low frequency, which can automatically adapt to the requirements of time-frequency signal analysis so that it can focus on any detail of the signal.

The time-domain function $\psi(t)$ corresponding to the function $\widehat{\psi}(\omega)$ satisfying the reconstruction condition $c_{\psi}=$ $\int w^{-1}|\widehat{\psi}(\omega)|^{2} \mathrm{~d} \omega<+\infty$ is called a wavelet basis function. $\psi(t)$ constitutes a function system by scaling and translation, and equation (10) is called a wavelet function system:

$$
\psi_{(a, b)}(t)=\frac{1}{\sqrt{|a|}} \psi\left(\frac{t-b}{a}\right)(b \in R, a \in R, a \neq 0),
$$

where $\psi_{(a, b)}(t)$ is called the subwavelet; $a$ is the scale factor expansion; and $b$ is the translation parameter.

The continuous wavelet transform of the signal sequence $f(t)=\in L^{2}(R)$ over time is defined as

$$
W_{f(a, b)}=\int_{-\infty}^{+\infty} f(t) \psi_{(a, b)}(t) \mathrm{d} t
$$

where $W_{f}(a, b)$ reacts to the frequency-domain characteristics through parameter $a$ and $b$ reflects the time-domain characteristics. It is the result of the discretization of the wavelet and the function $f(t)$ by the inner product operation and is also the similarity index between the sequence function $f(t)$ and the wavelet. If this index is large, the similarity is stronger; otherwise, it is weaker. The selection of wavelet basis functions needs to be combined with its properties, determined according to empirical methods, and the selection criteria are not uniform.

In [20], the authors pointed out that the wavelet transform solves the shortcomings of Fourier transform and short-time Fourier transform well. Fourier transform cannot automatically adjust the resolution of time domain and frequency domain according to the characteristics of the signal. The wavelet transform can analyze the local features of the signal, and its window size is variable. It has low entropy and multiresolution, which can remove the correlation of the signal. It can flexibly select the basis function according to the characteristics of the signal and is suitable for processing the unsteady signal of the motion positioning of the personnel in the tunnel.

3.2. Tunnel Positioning Signal Analysis. In this paper, the $\mathrm{db} 5$ wavelet basis function is used to analyze the signal motion of 
tunnel personnel. The support length of db5 wavelet basis function is 9 , the vanishing moment order is 5 , with approximate symmetry, regularity, and tight support. Wavelet analysis is performed on the $\mathrm{x}$-coordinate signal and the $y$-coordinate signal of the motion signal of the personnel in the tunnel, respectively, and the low-frequency coefficient and the high-frequency coefficient are separated and the influence of the motion noise on the positioning is studied. The target motion trajectory and the actual motion trajectory of the person moving in the tunnel are shown in Figure 3.

First, the signal is decomposed into five layers using the db5 wavelet basis function, and then, the low-frequency coefficients and high-frequency coefficients of the decomposed fifth layer to the first layer are reconstructed. The lowfrequency coefficients of the wavelet decomposition of the $\mathrm{x}$-coordinate signal are shown in Figure 4(a), and the highfrequency coefficients of the wavelet decomposition of the $\mathrm{x}$-coordinate signal are shown in Figure 4(b). The lowfrequency coefficients of the wavelet decomposition of the $y$-coordinate signal are shown in Figure 5(a), and the highfrequency coefficients of the wavelet decomposition of the $\mathrm{x}$-coordinate signal are shown in Figure 5(b).

Figure 4(a) consists of the original $x$-coordinate signal and the low-frequency coefficient reconstruction layer 1 to layer 5; Figure 4(b) consists of the original $x$-coordinate signal and the high-frequency coefficient reconstruction layer 1 to layer 5; Figure 5(a) consists of the original $y$ coordinate signal and the low-frequency coefficient reconstruction layer 1 to layer 5; Figure 5(b) consists of the original $y$-coordinate signal and the high-frequency coefficient reconstruction layer 1 to layer 5 .

As can be seen from Figures 4 and 5, the approximate signal of the $x$-coordinate signal can be obtained in the lowfrequency coefficient reconstruction layer 5 of Figure 4(a), and the approximate signal of the $y$-coordinate signal can be obtained in the low-frequency coefficient reconstruction layer 5 of Figure 5(a). In this layer, the influence of motion positioning noise on the signal can be neglected. The motion noise is mainly concentrated in high frequency noise, which can be processed by filtering and noise reduction. By comparing the Figures 4(b) and 5(b), it can be seen that the noise of the $x$-coordinate signal is significantly larger than the noise of the $y$-coordinate signal, and for the positioning of the human motion, the positioning error caused by the motion noise is within $\pm 50 \mathrm{~cm}$.

\section{Principle of Human Motion Positioning Noise Denoising}

The application of ultrawideband technology in tunnel personnel positioning faces many challenges, such as signal acquisition in real-world environments, multiuser mutual interference, multipath effects, and non-line-of-sight propagation. In practical applications, due to the diversity and irregularity of personnel movement, when using the UWB positioning system for positioning, a distance measurement result with abnormal results is generated. Therefore, the result of UWB personnel motion positioning

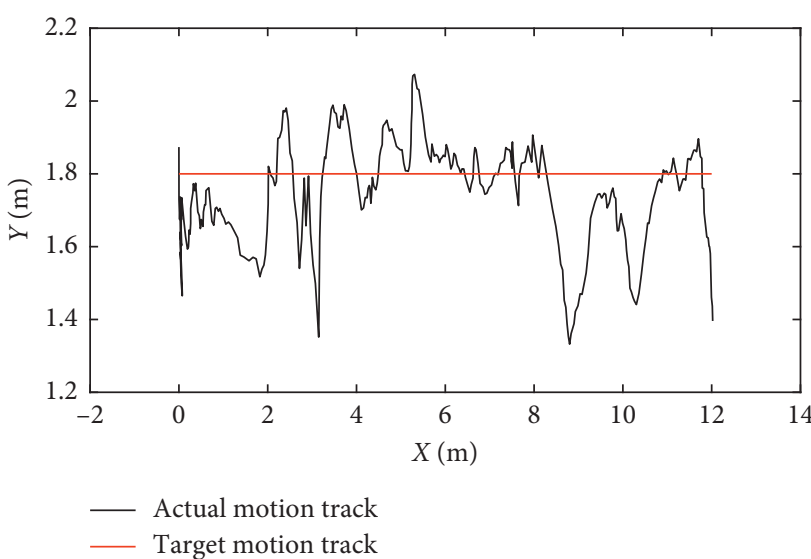

Figure 3: Personnel movement track.

is affected, and an abnormal jump point occurs, resulting in lower positioning accuracy and poor positioning reliability.

In this paper, the use of wavelet threshold denoising has the ability to highlight local features of signals in time and frequency domains. Through certain criteria, the detail component is identified, and the abnormal positioning result is extracted and zeroed. Then, reconstruct the original positioning result to achieve the purpose of eliminating the abnormal positioning point and smoothing the positioning result. It can solve the problem that the positioning of the tunnel personnel affects the positioning accuracy by positioning the abnormal point, weaken the influence of the positioning abnormal value, and obtain more accurate positioning results.

4.1. Wavelet Threshold Denoising. The traditional denoising methods mainly include linear filtering methods and nonlinear filtering methods, such as median filtering and Wiener filtering. Their disadvantages are that the entropy after signal transformation is increased, the nonstationary characteristics of the signal cannot be characterized, and the correlation of the signals cannot be obtained. In order to overcome the above disadvantages, wavelet transform is used to solve the denoising problem. The method of wavelet threshold denoising has the advantages of simple calculation, easy implementation, and good effect.

The process of wavelet threshold denoising is shown in Figure 6.

First, select a wavelet basis function, determine the level of decomposition and then perform the decomposition calculation. Different wavelet basis functions have different characteristics in signal processing. When denoising different types of signals, wavelet basis functions should be selected according to experience and the selection criteria are inconsistent. In the previous study of this paper, we chose the wavelet basis function. Three kinds of wavelet basis functions, db5, sym5, and coif5, were used to denoise the results of UWB indoor human motion positioning and to compare the denoising effect. The experimental results show that the db5 wavelet basis function is more suitable for denoising the results of UWB human motion positioning, and the denoising effect is the best. Therefore, this paper 
The original $x$-coordinate signal

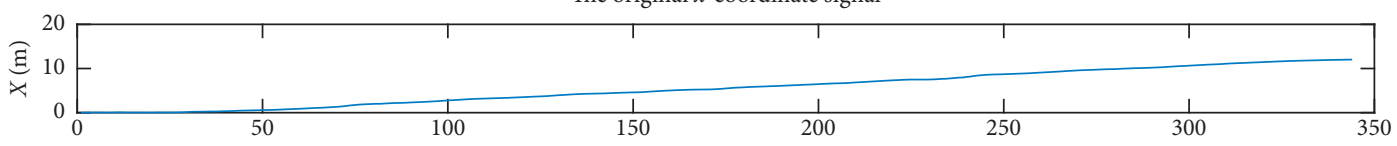

Low-frequency coefficient reconstruction layer 5

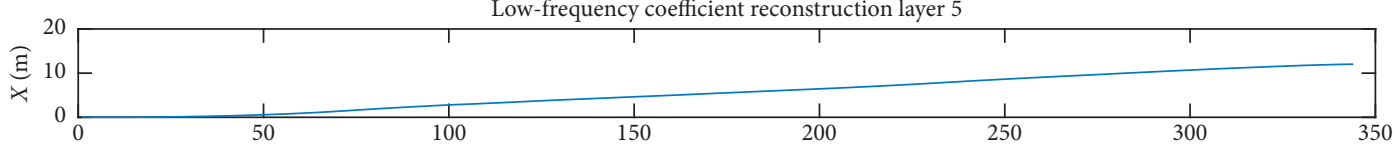

Low-frequency coefficient reconstruction layer 4

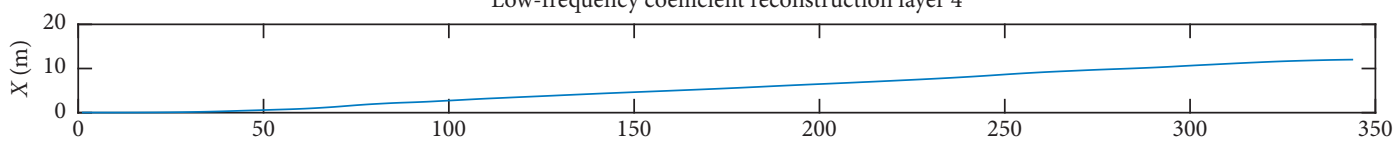

Low-frequency coefficient reconstruction layer 3
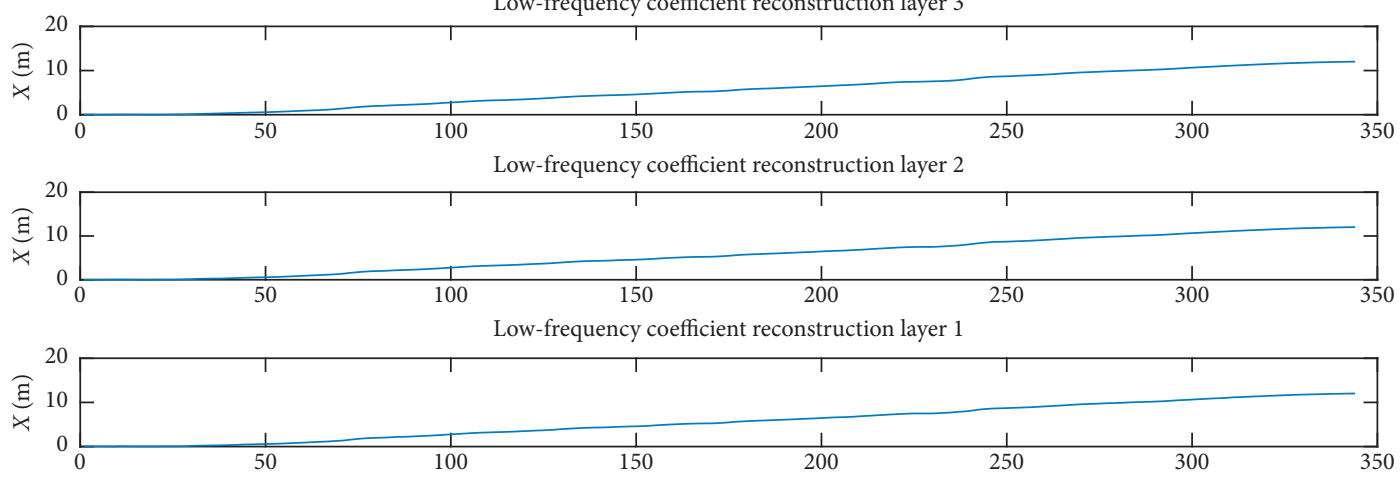

(a)

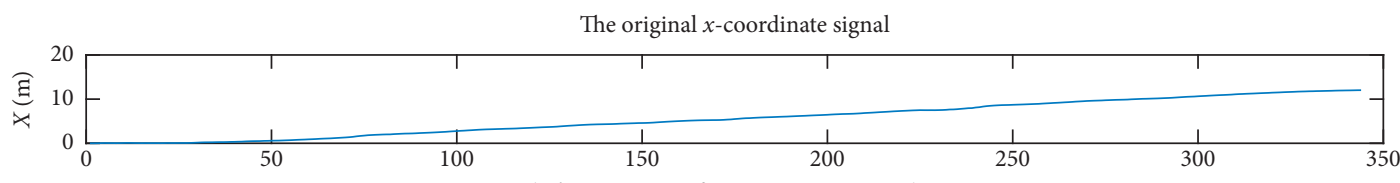

High-frequency coefficient reconstruction layer 5

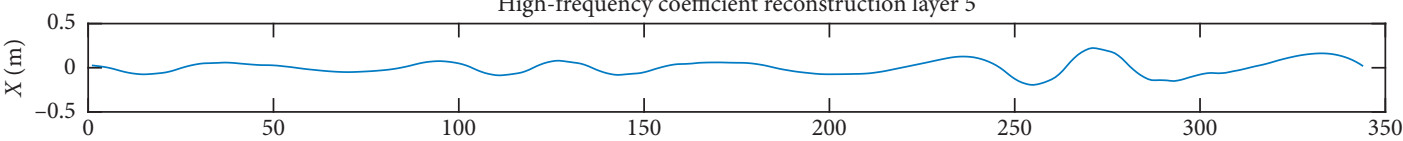

High-frequency coefficient reconstruction layer 4

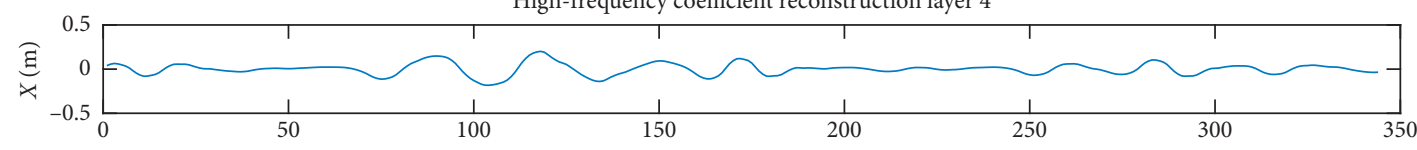

High-frequency coefficient reconstruction layer 3

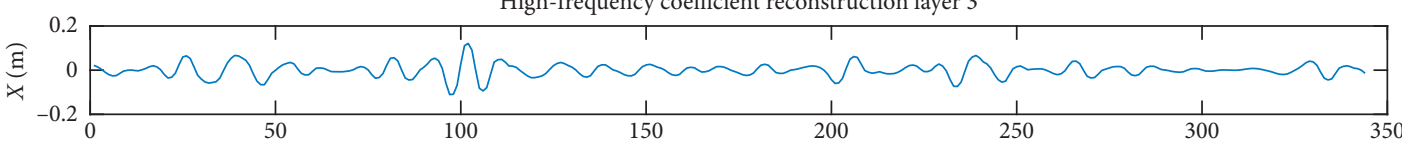

High-frequency coefficient reconstruction layer 2
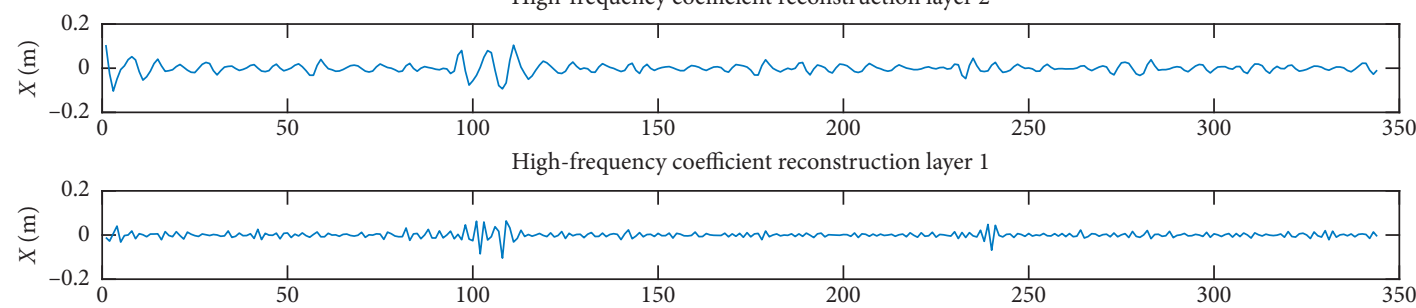

(b)

FIgURE 4: (a) Low-frequency coefficient of wavelet decomposition of $x$-coordinate signal. (b) High-frequency coefficient of wavelet decomposition of $x$-coordinate signal. 

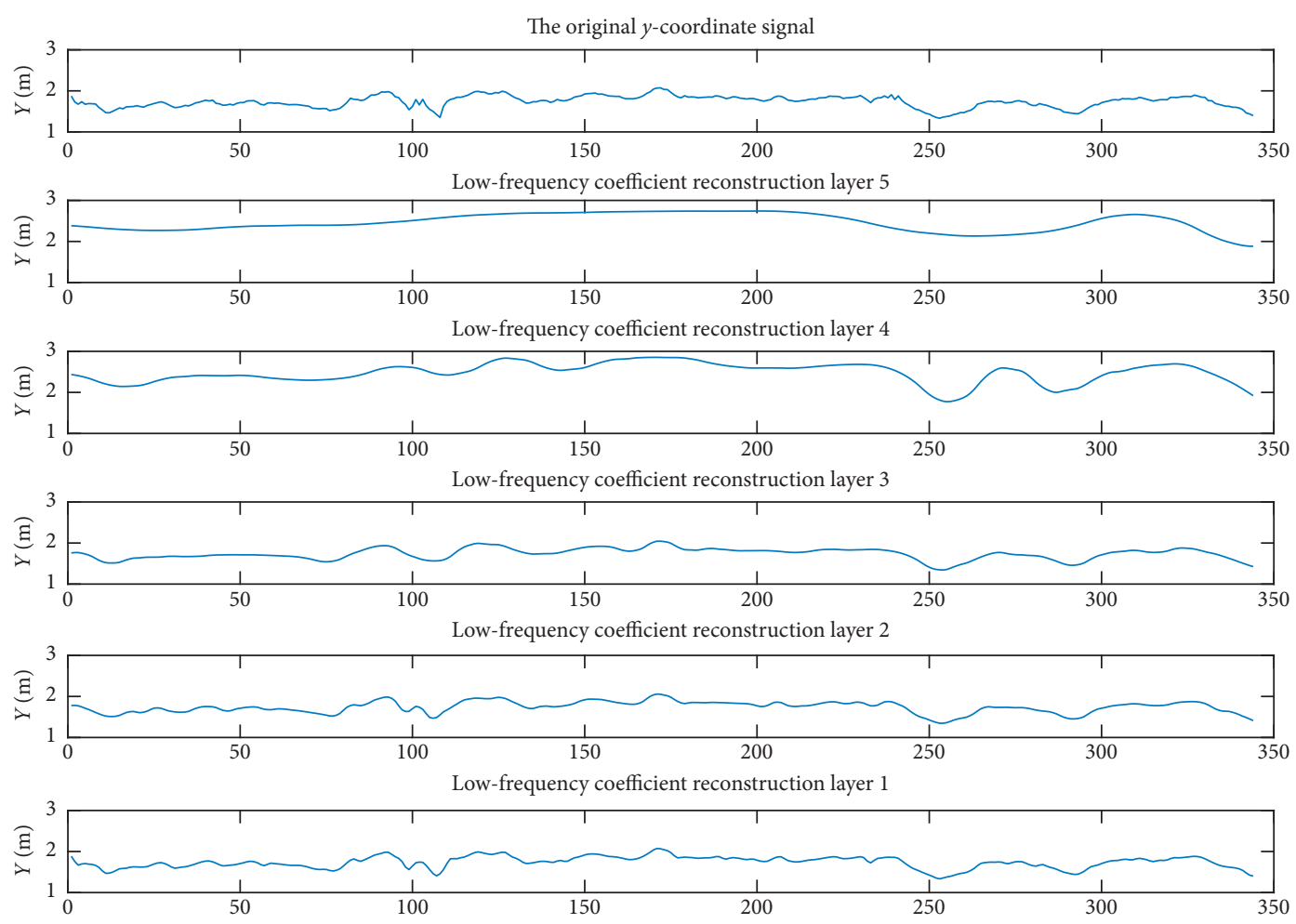

(a)

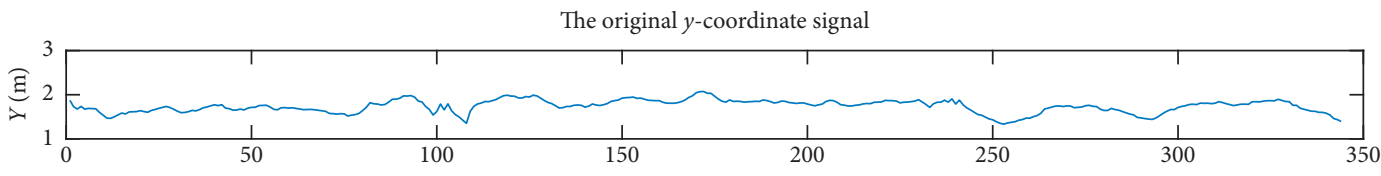

High-frequency coefficient reconstruction layer 5

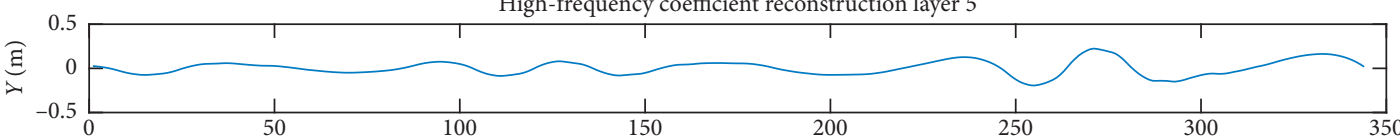

High-frequency coefficient reconstruction layer 4

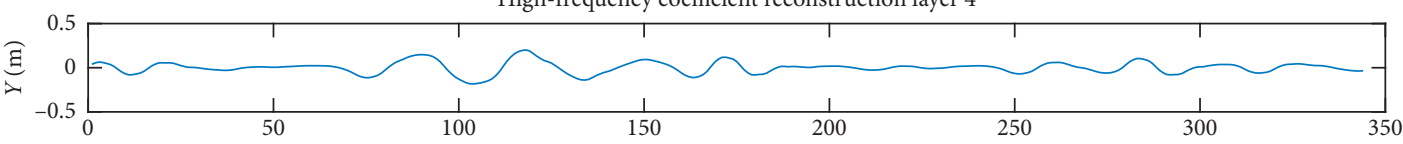

High-frequency coefficient reconstruction layer 3

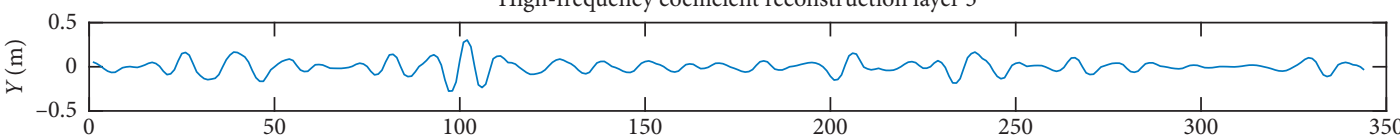

High-frequency coefficient reconstruction layer 2
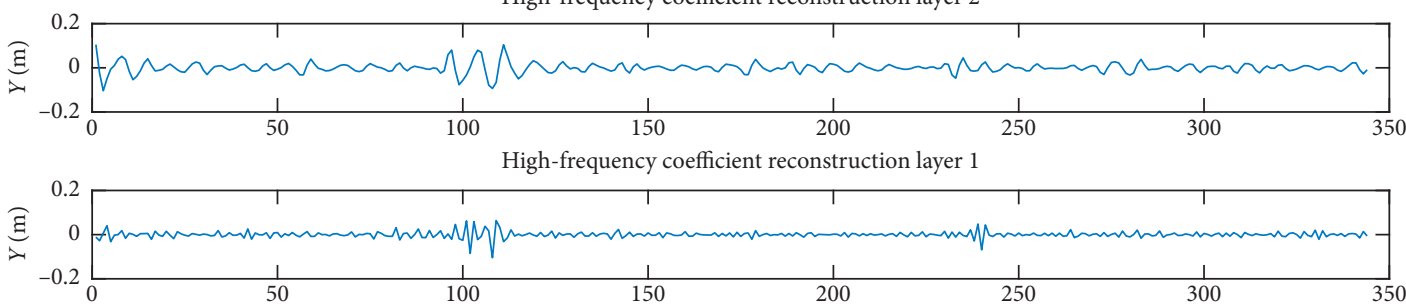

(b)

FIGURE 5: (a) Low-frequency coefficient of wavelet decomposition of $y$-coordinate signal. (b) High-frequency coefficient of wavelet decomposition of $y$-coordinate signal. 


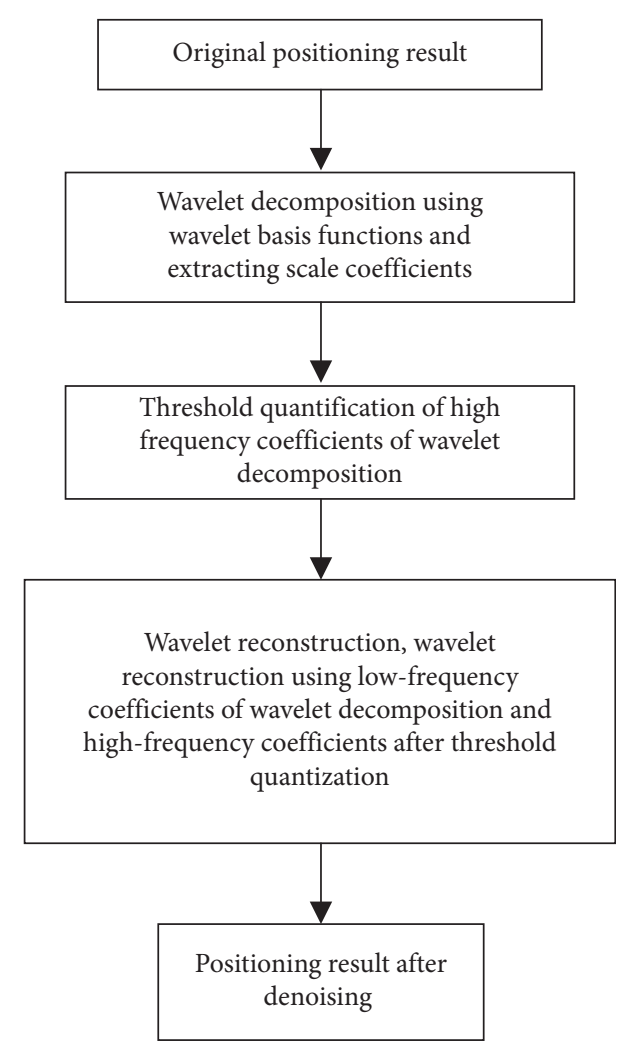

FIGURE 6: Wavelet threshold denoising process.

continues to choose db5 wavelet basis function for wavelet threshold denoising.

A schematic diagram of the structure of the tower decomposition algorithm for wavelet decomposition of the original positioning result is shown in Figure 7 . Then, a threshold is selected for the high-frequency coefficients of each decomposition scale to be quantized. Finally, the lowest frequency coefficient of wavelet decomposition and the high frequency coefficient of each layer are used for wavelet reconstruction.

Suppose that $H$ and $G$ are low-pass filters and high-pass filters, respectively. $C_{\mathrm{j}}$ and $D_{\mathrm{j}}$ are the approximate and detail components of the original signal at resolution $2-j$, respectively. Then, we can get

$$
\left\{\begin{array}{l}
C_{j+1}=H C_{j}, \\
D_{j+1}=G C_{j}, \\
j=1,2,3,4,5 .
\end{array}\right.
$$

Suppose $H^{*}$ and $G^{*}$ be the dual operators of $H$ and $G$ respectively, then we can get:

$$
C_{j}=H^{*} C_{j+1}+G^{*} D_{j+1} \text {. }
$$

It can be seen from the decomposition and reconstruction of the wavelet that the wavelet transform is equivalent to the group filter, the approximate component is the low-frequency component of the original positioning result, and the detail component corresponds to the highfrequency component of the original positioning result. The anomaly positioning point in the original positioning result corresponds to the high-frequency component, so the data of the abnormal positioning after the wavelet decomposition should be included in the detail component of the wavelet decomposition. After the wavelet decomposition, the detail component is observed, the detail component containing the abnormal positioning point is set to zero, and then the reconstructed signal can achieve the purpose of eliminating the abnormal positioning point.

A schematic diagram of the structure of the tower reconstruction algorithm for wavelet reconstruction of the original positioning result is shown in Figure 8.

The wavelet reconstruction of the original positioning result is calculated according to the low frequency coefficient of the fifth layer of the wavelet decomposition and the modified high-frequency coefficient from the first layer to the fifth layer. Finally, the detuned tunneling motion positioning result is obtained, the abnormal value in the positioning result is removed, and the positioning accuracy are improved.

4.2. Choice of Threshold Function. The threshold selection rule is based on a model $Y=f(t)+e$, where $e$ is a Gaussian white noise $N(0,1)$ and $f(t)$ is the original signal. Evaluation by wavelet coefficients, or raw signals, can eliminate the threshold of noise in the wavelet domain. The heuristic threshold estimation is more thorough in denoising and more effective in denoising. Therefore, the heuristic threshold selection method is adopted in this paper.

After determining the threshold of the wavelet coefficient of the Gaussian white noise, a threshold function is needed to filter the wavelet coefficient containing the noise coefficient to remove the Gaussian noise figure. Donoho divides the threshold processing into a soft threshold and a hard threshold. Suppose $w$ be the magnitude of the wavelet coefficient, $w_{\lambda}$ be the size of the wavelet coefficient after the threshold is applied, and $\lambda$ be the threshold.

For the hard threshold denoising method, when the absolute value of the wavelet coefficient is less than a given threshold, let it be zero. When it is greater than the threshold, keep it unchanged, which can be expressed as

$$
w_{\lambda}= \begin{cases}w, & |w|>\lambda \\ 0, & |w|<\lambda\end{cases}
$$

For the soft threshold denoising method, when the absolute value of the wavelet coefficient is less than a given threshold, let it be zero. When it is greater than the threshold, let it subtract the threshold, which can be expressed as

$$
w_{\lambda}= \begin{cases}{[\operatorname{sign}(w)](|w|-\lambda),} & |w|>\lambda, \\ 0, & |w|<\lambda .\end{cases}
$$

The hard threshold function is superior to the soft threshold method in the sense of the mean square error, but the signal will generate additional oscillations, generate jump points, and have no smoothness of the original signal. The overall stability of the wavelet coefficients obtained by the soft threshold estimation is better, so that the estimated 


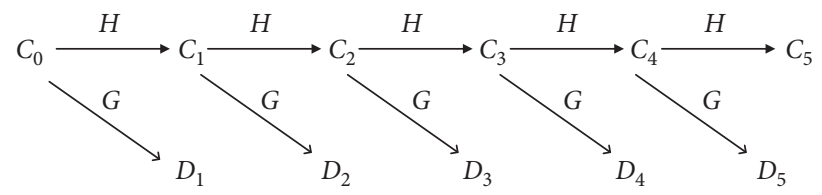

FIgURE 7: Schematic diagram of wavelet tower decomposition algorithm.

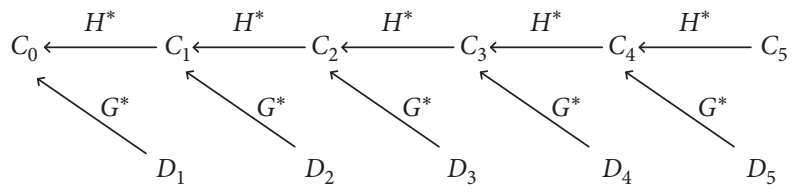

FIgURE 8: Schematic diagram of wavelet tower reconstruction algorithm.

signal does not generate additional oscillation, but it is better than the compressed signal, which will produce a certain deviation, which directly affects the degree of approximation of the reconstructed signal and the real signal.

In the following chapter, the soft threshold function and the hard threshold function are selected, respectively, to perform wavelet threshold processing on the motion positioning result of the personnel in the tunnel. Analyze which threshold function is more suitable for the positioning of people in the tunnel.

\section{Experiment and Analysis}

In order to verify the influence of the tunnel positioning method on the positioning accuracy of the UWB positioning system, two sets of experiments were designed, which were the static positioning experiment and the personnel motion positioning experiment. The static experiment is mainly used to confirm the influence of the tunnel environment on the positioning accuracy, and the human motion positioning experiment is used to observe the motion noise removal effect.

Due to the limitations of the experimental environment, the experiments involved in this paper can no longer be measured in the tunnel environment under construction and the actual tunnel environment is replaced by the measurement in the underground channel. The experimental content includes the following two items: (1) personnel static positioning experiment, testing the accuracy of the positioning system; (2) personnel motion positioning experiment; the wavelet denoising is performed on the positioning result, and the soft threshold function and the hard threshold function are, respectively, used to determine a method suitable for the motion positioning of the personnel in the tunnel to eliminate the motion noise.

5.1. Experimental Environment and Equipment. The location of the experiment in this paper was selected to be an underground passage of $4 \mathrm{~m} \times 35 \mathrm{~m} \times 2.5 \mathrm{~m}$, as shown in Figure 9(a). The ultrawideband positioning system based on DW1000 chip is used in the experimental equipment. The
DW1000 chip is a chip launched by Decawave in 2012. It is based on ultrawideband technology and conforms to the IEEE802.15.4-2011 standard. Decawave's DWM1000 module integrates the DW1000 chip and ceramic antenna on one module. Although it is convenient to use, there are some shortcomings in actual use. For example, because the orientation of the ceramic antenna is not good, the direction of the module antenna is different, and the ranging result is different, which affects the positioning accuracy. Moreover, the gain of the ceramic antenna is too low, only about $-3 \mathrm{db}$, resulting in the module's communication distance to be too close, only 30-50 meters.

In response to these problems, we have designed a new ultrawideband module based on DW1000 chip, which contains DW1000 chip and SMA connector, which can connect external antenna with better performance and make up for the shortcomings of the DWM module's poor directivity, close distance and so on. The external antenna used in this experiment is the rod antenna GX030592RS, which has the advantages of high gain and long distance. At $110 \mathrm{k}$ baud rate and 1024 preamble length configuration, the measured distance can be 400 meters, which is suitable for personnel positioning in the tunnel. The experimental device, as shown in Figure 9(b), includes three positioning base stations and one tag.

The base stations $A 0, A 1$, and $A 2$ are arranged in the tunnel, and the coordinates of the three positioning base stations are set to $A 0(0,0), A 1(12,0)$, and $A 2(6,3)$, and the unit is $\mathrm{m} . A 0$ is a communication base station, and serial output is performed on the computer. At the same time, in order to effectively monitor the data in the system, the monitoring software interface shown in Figure 9(c) is used.

5.2. Experiment of Static Positioning of Personnel. In this experiment, the positioning experiment was carried out at six locations while the person was stationary. The positioning results of 6 different points were analyzed. The positioning experiment of each point lasted for 1 minute, and about 600 position coordinates were obtained. The test point settings of the personnel static positioning experiment are shown in Table 1 and Figure 10(a). 


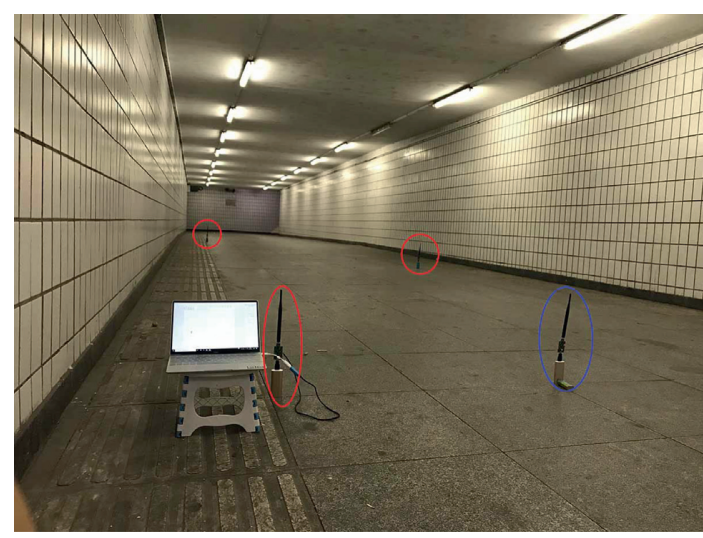

(a)

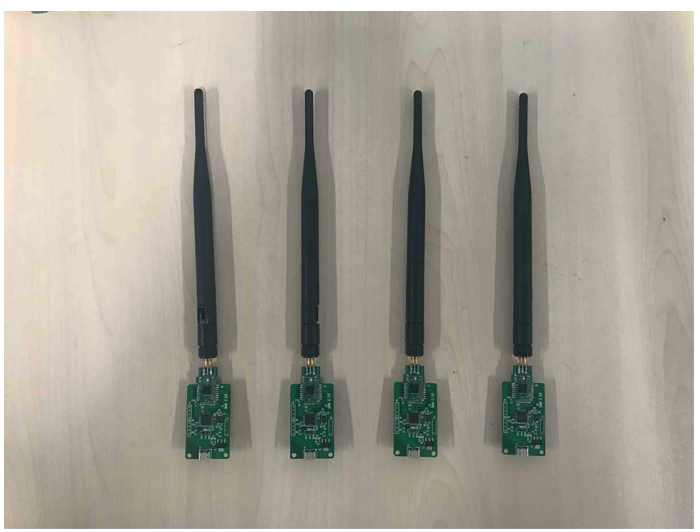

(b)

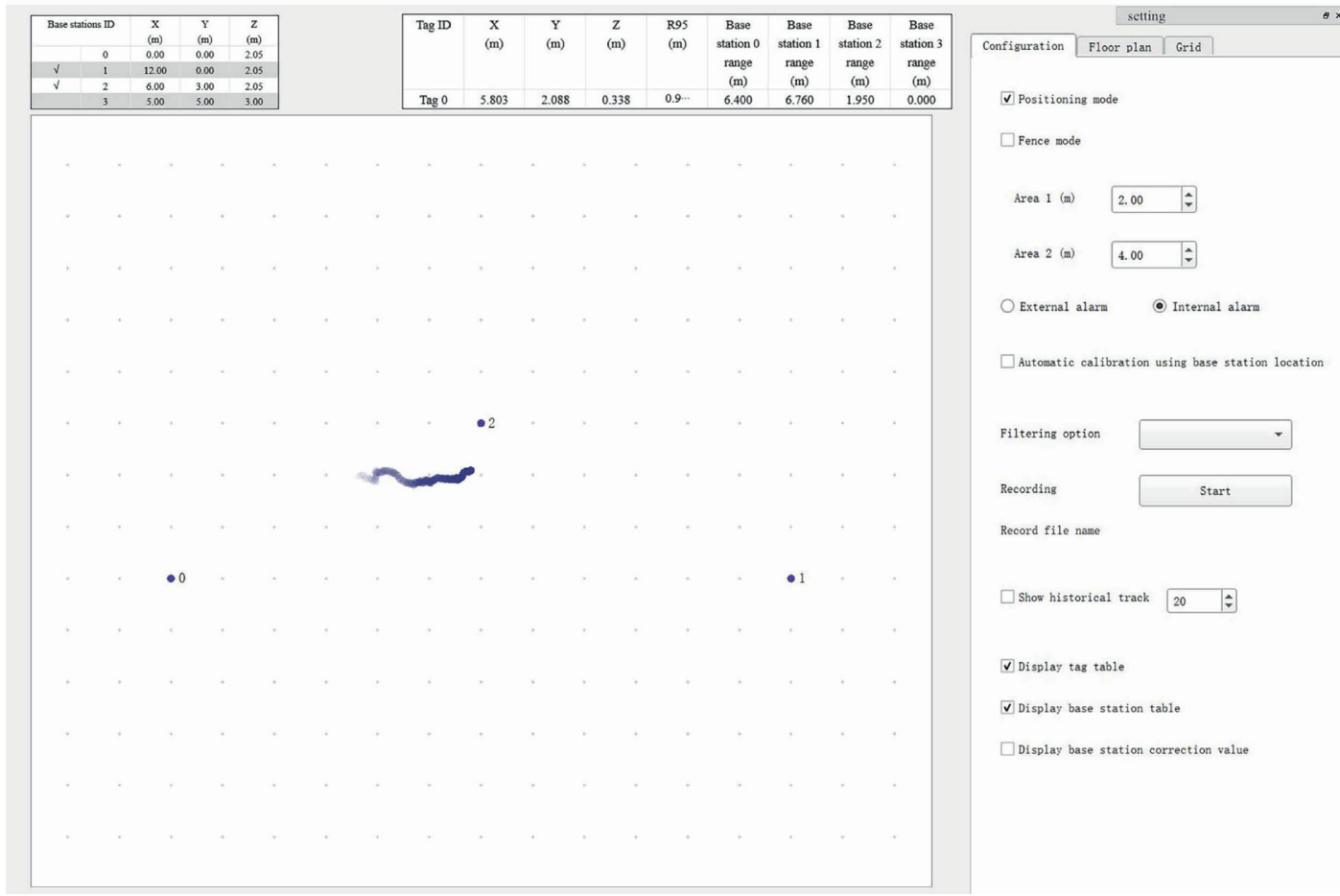

(c)

FIgURE 9: (a) Experimental test environment. (b) Experimental equipment. (c) Monitoring software interface.

TABle 1: Coordinate of test point.

\begin{tabular}{lcc}
\hline Test point & $X$-coordinate value $(\mathrm{m})$ & $Y$-coordinate value $(\mathrm{m})$ \\
\hline T1 & 0 & 1.81 \\
T2 & 2.41 & 1.81 \\
T3 & 4.82 & 1.81 \\
T4 & 7.23 & 1.81 \\
T5 & 9.64 & 1.81 \\
T6 & 12 & 1.81 \\
\hline
\end{tabular}

The positioning is performed by the trilateral positioning method, and the result of the static positioning of the personnel is as shown in Figure 10(b).

The positioning is performed by the three-side positioning method, and the accuracy of the positioning results of different points is as shown in Table 2 .
It can be seen from Figure 10(b) and Table 2 that in the static positioning experiment of the personnel, compared with the real position, after positioning using the three-sided positioning method, the average positioning error of the six test points is about $18 \mathrm{~cm}$ and the maximum positioning error is $31 \mathrm{~cm}$. Therefore, the triangulation method can be used to locate the tunnel personnel to meet the needs of the tunnel personnel.

5.3. Experiment of Human Motion Positioning. The experiment was tested in the case of personnel movement. The tester wears the UWB positioning tag and walks according to the test trajectory shown in Figure 11.

The original test trajectory of the human motion localization is derived, and then, the $\mathrm{db} 5$ wavelet basis function 


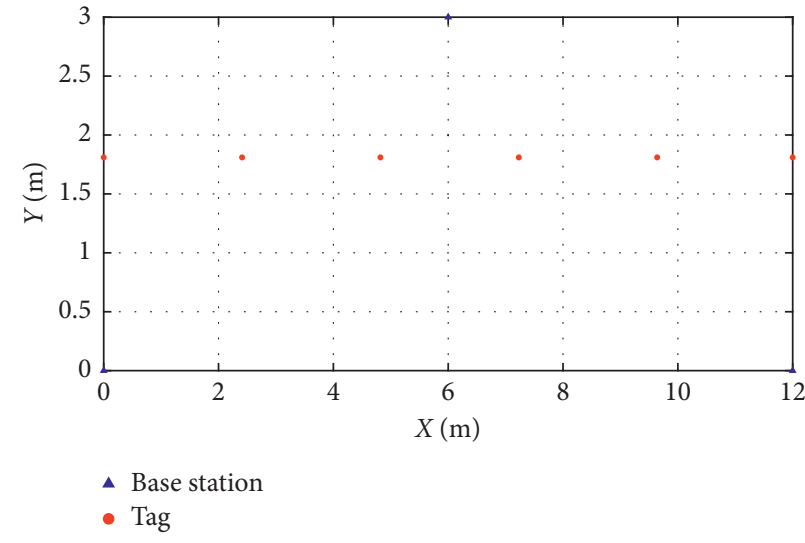

(a)

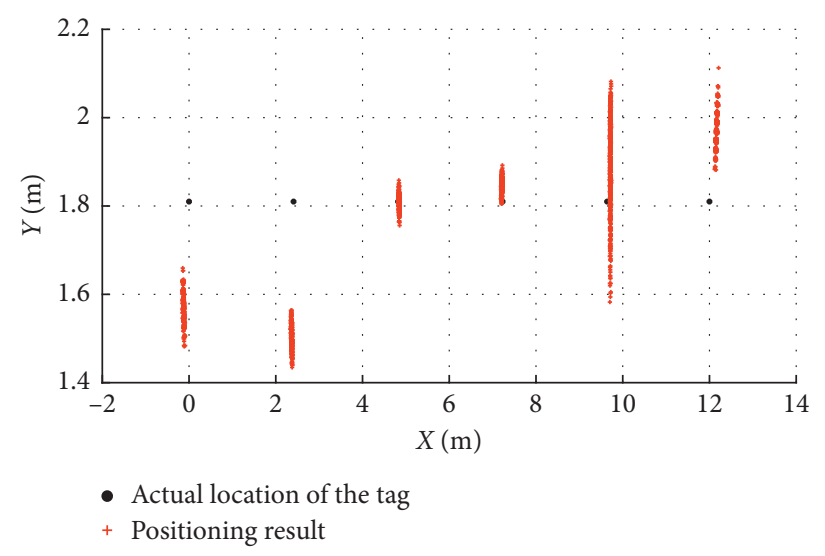

(b)

Figure 10: (a) Plane position distribution map of test points. (b) Positioning result of the trilateral positioning method.

TABLE 2: Accuracy statistics of personnel static positioning results.

\begin{tabular}{lcc}
\hline Test point & Standard deviation $(\mathrm{cm})$ & Root mean square error $(\mathrm{cm})$ \\
\hline T1 & 3.21 & 27.53 \\
T2 & 2.50 & 31.13 \\
T3 & 1.76 & 2.95 \\
T4 & 1.63 & 4.29 \\
T5 & 10.45 & 15.14 \\
T6 & 3.54 & 23.21 \\
Average value & 3.85 & 17.37 \\
\hline
\end{tabular}

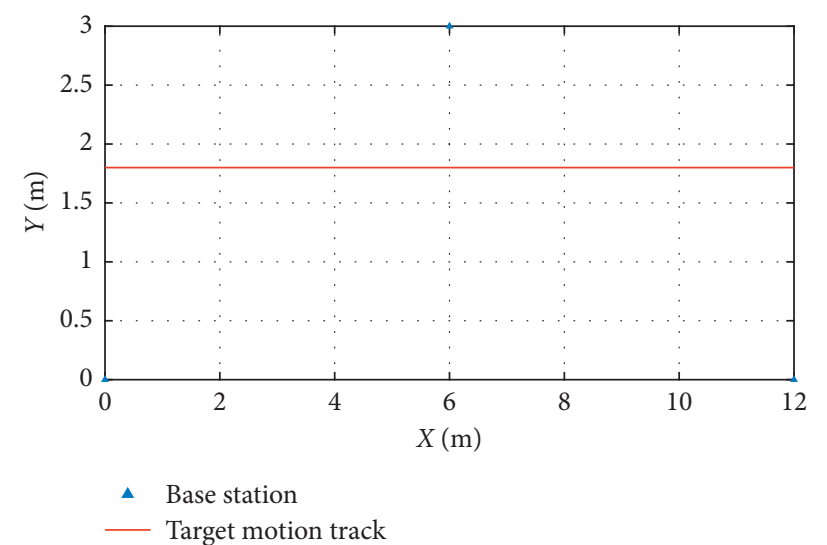

FIGURE 11: Test track for human motion positioning.

and the heuristic threshold selection are used, and the soft threshold function and the hard threshold function are, respectively, used for denoising. The result of denoising the original trajectory and the wavelet threshold by using the soft threshold function to locate the motion of the person in the tunnel is shown in Figure 12(a). The result of denoising the original trajectory and the wavelet threshold by using the hard threshold function to locate the motion of the person in the tunnel is shown in Figure 12(b).

It can be seen from Figures 12(a) and 12(b) that the experimental trajectory of the human motion positioning is closer to the test trajectory after wavelet denoising. And by comparison, it can be seen that the soft threshold function is more suitable for the wavelet threshold denoising method for the motion positioning of people in the tunnel. It has the best effect of removing the abnormal value and smoothing the target trajectory during the movement of the personnel and will not lose useful information. After the wavelet threshold denoising, the maximum error has been changed from $70 \mathrm{~cm}$ to $40 \mathrm{~cm}$, effectively reducing the error caused by the movement of people.

Finally, in order to ensure the accuracy of the experiment, the human motion positioning experiment in the tunnel was performed again. The tester follows the test trajectory of the walk as a rectangle to ensure that the method can also be applied when the trajectory of the person 


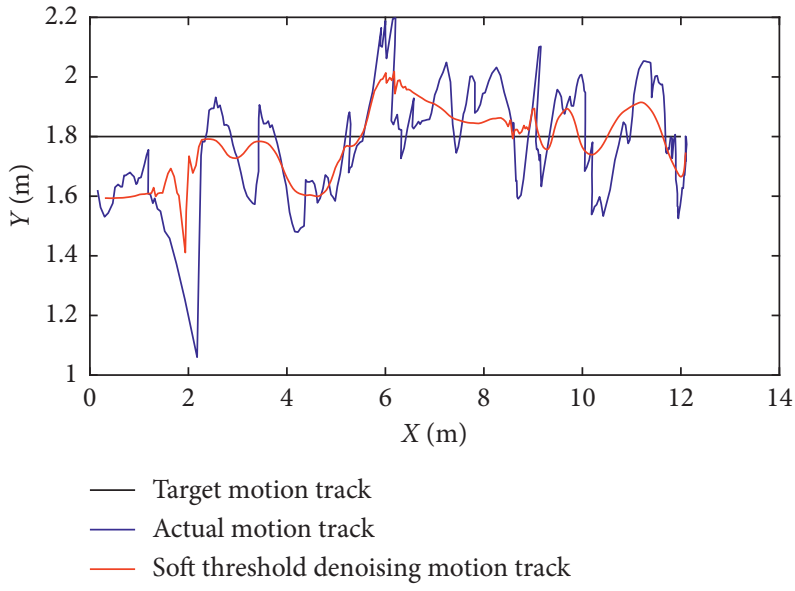

(a)

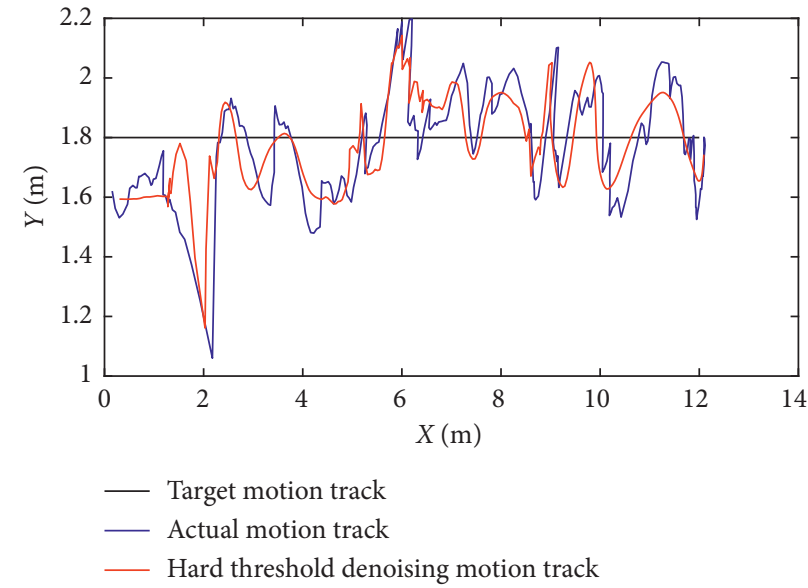

(b)

Figure 12: (a) The soft threshold function for human motion positioning results before and after wavelet denoising. (b) The hard threshold function for human motion positioning results before and after wavelet denoising.

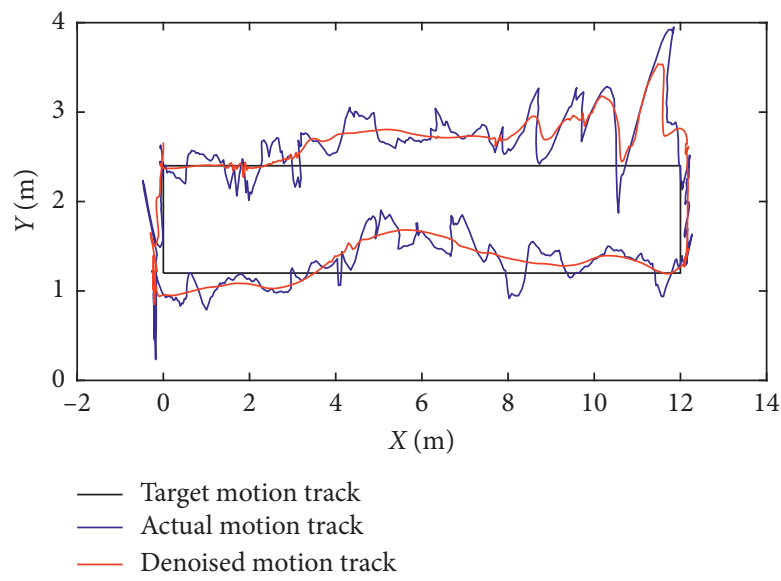

(a)

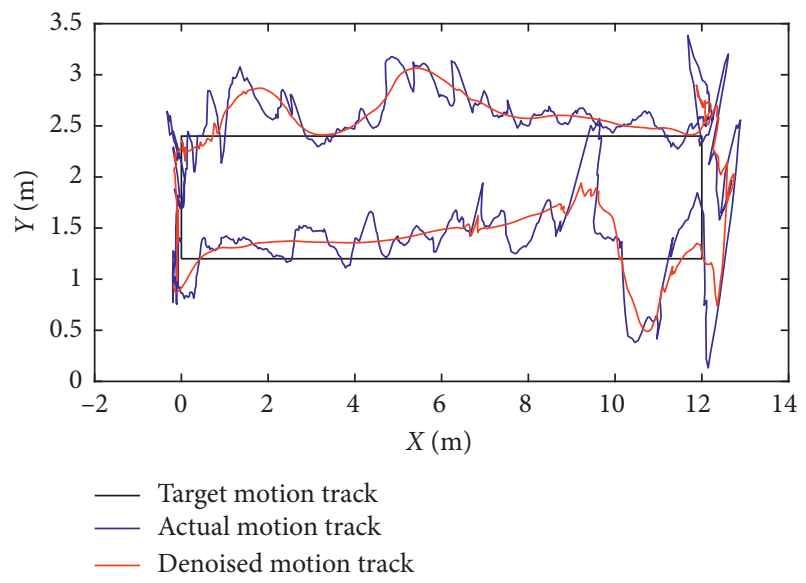

(b)

Figure 13: Personnel motion localization results before and after wavelet denoising: (a) first group; (b) second group.

is different. The two test results were denoised by the wavelet threshold according to the optimal denoising scheme, and the results are shown in Figures 13(a) and 13(b).

It can be seen from Figure 13 that the actual motion trajectory of the human motion positioning is closer to the test trajectory after wavelet denoising. It is proved that the wavelet threshold denoising method can be used in different motion trajectories, which can weaken the influence of abnormal positioning points caused by human motion noise and make the motion trajectory of personnel more smooth, and the effective positioning information is not lost in the process of denoising.

\section{Conclusion}

In recent years, the tunnel construction of highway, railway, and subway as an important part of traffic network is developing constantly and the safety of tunnel construction has become a hot issue. In the process of tunnel construction, the real-time safety monitoring of the tunnel construction site and obtaining the specific location information of each construction personnel are of great significance to reduce the loss of accidents, and the accurate positioning of the tunnel cannot be ignored.

According to the requirements and characteristics of tunnel location, this paper uses UWB technology to measure distance using the symmetric double-sided two-way ranging method and locates it by TOA-based trilateral positioning algorithm. Through the static positioning experiment of the personnel in the tunnel, it is proved that the positioning error of the positioning algorithm is within $20 \mathrm{~cm}$, which is suitable for the positioning requirements of tunnel personnel.

In the actual experiment, we found that due to the diversity and irregularity of the movement of the people, the noise of the motion was generated and the noise could not be completely avoided. When using the UWB positioning system to locate people in motion, the positioning results 
will have abnormal jump points and fluctuations. As a result, the positioning accuracy is lowered and the positioning reliability is deteriorated.

We perform wavelet analysis on the motion positioning signal of the personnel in the tunnel and determine the influence of the motion noise of the personnel on the UWB positioning by separating the low-frequency coefficient and the high-frequency coefficient of the signal. The method of wavelet threshold denoising is used to process the motionlocating trajectory of ultra-wideband personnel. The soft threshold function and the hard threshold function are, respectively, selected to determine the wavelet threshold denoising method which is more suitable for the motion location of the personnel in the tunnel. It is verified by the human motion positioning experiment in the tunnel. Compared with the hard threshold function, the wavelet threshold denoising method using the soft threshold function is more suitable for the motion positioning of the personnel in the tunnel. The experimental results show that the method is suitable for tunnel personnel positioning, which can weaken the influence of personnel motion on the UWB positioning, eliminate the abnormal positioning point, and smoothen the positioning result.

\section{Data Availability}

The attachment data used to support the findings of this study are included within the article.

\section{Conflicts of Interest}

The authors declare that they have no conflicts of interest.

\section{Authors' Contributions}

Ning Liu and Ranqiao Zhang contributed equally to this work.

\section{Acknowledgments}

This work was supported by the National Natural Science Foundation of China (61801032), the Beijing Natural Science Foundation (3184046), General Project of Science and Technology Program of Beijing Education Commission (77F1910963), and the Beijing Key Laboratory of High Dynamic Navigation Technology, Qin Xin Talents Cultivation Program, Beijing Information Science \& Technology University, and Laboratory of Modern Measurement \& Control Technology (Beijing Information Science \& Technology University Ministry of Education).

\section{References}

[1] T. Xu, S. Cao, W. Zhou et al., "Design and study of personnel positioning tracking system for long tunnel construction," Highway, vol. 59, no. 2, pp. 113-117, 2014.

[2] R. Wang and X. Xi, "Application of improved LANDMARC algorithm in tunnel personnel location," Journal of the China Railway Society, vol. 38, no. 1, pp. 70-74, 2016.

[3] O. Yang, "Improved locating algorithm of railway tunnel personnel based on collaborative information fusion in
Internet of Things," Transactions of the Institute of Measurement and Control, vol. 39, no. 4, pp. 446-454, 2017.

[4] C. Qi, F. Amato, M. Alhassoun et al., "Breaking the range limit of RFID localization: phase-based positioning with tunneling tags," in Proceedings of the IEEE International Conference on RFID, Phoenix, AZ, USA, April 2019.

[5] H. Wang, C. Run, X. Chang et al., "Research on accuracy of tunnel personnel positioning based on ZigBee technology," Quality Measurement and Control, vol. 33, no. 5, pp. 111-114, 2016.

[6] M. Cypriani, G. Delisle, and N. Hakem, "Wi-Fi-based positioning in underground mine tunnels," in Proceedings of the International Conference on Indoor Positioning \& Indoor Navigation, IEEE, Montbeliard, France, October 2013.

[7] W. Fang and Y. Gao, "A weighted centroid localization algorithm based on RSSI for wireless sensor network in straight narrow tunnel," Chinese Journal of Sensors and Actuators, vol. 27, no. 2, pp. 247-251, 2014.

[8] R. A. Fayadh, F. Malek, and H. A. Fadhil, "Pulse sign separation technique for the received bits in wireless ultrawideband combination approach," Mathematical Problems in Engineering, vol. 2014, Article ID 919681, 10 pages, 2014.

[9] D. Kocur, J. Fortes, and M. Svecova, "Multiple moving person tracking by UWB sensors: the effect of mutual shielding persons and methods reducing its impacts," Eurasip Journal on Wireless Communications and Networking, vol. 2017, no. 1, pp. 68-83, 2017.

[10] S. Li, G. Li, L. Wang, Y. Zhou, Y. Peng, and J. Fu, "A threedimensional robust ridge estimation positioning method for UWB in a complex environment," Advances in Space Research, vol. 60, no. 12, pp. 2763-2775, 2017.

[11] Y. Sun and Y. Dai, "System design and algorithm research of UWB wireless location," Science Technology and Engineering, vol. 16, no. 31, pp. 202-205, 2016.

[12] R. A. Scholtz, "Multiple access with time-hopping impulse modulation," in Proceedings of the MILCOM'93-IEEE Military Communications Conference, pp. 447-450, Boston, MA, USA, October 1993.

[13] V.-H. Nguyen and J.-Y. Pyun, "Location detection and tracking of moving targets by a 2D IR-UWB radar system," Sensors, vol. 15, no. 3, pp. 6740-6762, 2015.

[14] Y. Xu, Y. S. Shmaliy, Y. Li, and X. Chen, "UWB-based indoor human localization with time-delayed data using EFIR filtering," IEEE Access, vol. 5, pp. 16676-16683, 2017.

[15] V. Savic, J. Ferrer-Coll, P. Angskog, J. Chilo, P. Stenumgaard, and E. G. Larsson, "Measurement analysis and channel modeling for TOA-based ranging in tunnels," IEEE Transactions on Wireless Communications, vol. 14, no. 1, pp. 456467, 2015.

[16] M. Ridolfi, S. Vandermeeren, J. Defraye et al., "Experimental evaluation of UWB indoor positioning for sport postures," Sensors, vol. 18, no. 1, p. 168, 2018.

[17] C. Wang, J. Wang, Y. Ning et al., "Study of noise reduction method for ultra wideband positioning," Science of Surveying and Mapping, vol. 4, pp. 175-181, 2019.

[18] Y. Peng, Wavelet Analysis in One-Dimensional Signal Denoising, Beijing University of Posts and Telecommunications, Beijing, China, 2011.

[19] C. Lin, X. Qin, and Z. Jia, "Research of UWB indoor location based on wavelet analysis and full centroid position scheme," Computer Simulation, vol. 31, no. 2, pp. 391-395, 2014.

[20] P. Gong, Y. Mao, and Y. Du, "An UWB indoor location algorithm based on wavelet de-noising," Microcomputer and its Applications, vol. 34, no. 10, pp. 61-63, 2015. 
[21] Z. Shan, J. Zhou, and J. Chen, "Background noise suppression of magnetic anomaly signal based on wavelet transform," in Proceedings of the IEEE International Conference on Communication Technology, pp. 333-337, IEEE, Chongqing, China, October 2018.

[22] A. Kumar, R. Komaragiri, and M. Kumar, "Heart rate monitoring and therapeutic devices: a wavelet transform based approach for the modeling and classification of congestive heart failure," ISA Transactions, vol. 79, pp. 239-250, 2018.

[23] Z. Li, Y. Zong, and Y. Shi, "Application of wavelet threshold denoising method in signal processing of deep small hole drilling acoustic emission," Test and Quality, vol. 9, pp. 113-117, 2019.

[24] Y. Zhang, Health Monitoring System of Tunnel Structure Based on UWB, Dalian University of Technology, Dalian, China, 2018. 